\section{Bone marrow niches of germline FANCC/FANCG deficient mice enable efficient and durable engraftment of hematopoietic stem cells after transplantation}

Efficient and durable hematopoietic stem cell (HSC) engraftment is essential for successful HSC transplantation (HSCT). Multiple previous studies had demonstrated active participation of specialized bone marrow (BM) niches in regulating HSC engraftment during HSCT. $^{1-3}$ Recently, studies using models of human inherited BM failure syndromes (iBMFS) have demonstrated that BM niche dysfunction contributes to BM failure (BMF) pathogenesis and HSC loss. ${ }^{4-7}$ However, whether impaired niche cell survival or function diminishes healthy donor HSC engraftment following curative HSCT for these iBMFS remains undefined. Fanconi anemia (FA) is a BMF associated with germline mutations in one of 23 known FA genes that contribute to intrastrand DNA crosslinks and homologous recombination. ${ }^{8,9}$ Several mouse strains with germline FA gene deletion have been established during the last decade. ${ }^{10}$ Specifically, Fancc Fanc $^{-1 /}$ double knockout mice have been described as developing BMF spontaneously. ${ }^{11}$ Reduction in HSC has now also been described in mice lacking FANCG alone. ${ }^{12}$

A recent study in this journal explored whether functional defects in mesenchymal stem/progenitor cells
(MSPC) from BM niches of $\mathrm{Fancc}^{-/} \mathrm{Fancg}^{-/}$mice contribute to BMF development. ${ }^{5}$ However, it is still not clear whether germline deficiency of FA genes within MSPC impairs the capacity of recipient BM niches to engraft functionally normal donor HSC post transplantation. Here we demonstrate that BM niches of mice with germline deficiency of FANCC and/or FANCG remain capable of efficient and durable engraftment of donor HSC post HSCT.

Given that BMF arises in FA following exposure to environmental stress, ${ }^{13}$ we first analyzed whether

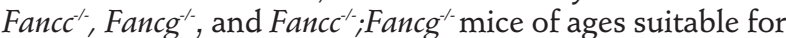
HSCT developed BMF. None of these FA models demonstrated deficiency in peripheral blood leukocyte, neutrophil, hemoglobin or platelet counts compared with wild-type (WT) mice up to 40 weeks of age (Figure 1A). BM cellularity was also not reduced in $\mathrm{FanCc}^{-1}$; $\mathrm{Fancg}^{-}$ versus WT mice up to 40 weeks of age (Figure $1 \mathrm{~B}$ and $\mathrm{C}$ ). Quantification of $\mathrm{HSC} /$ progenitor subsets showed no changes in percentages of LSK $\left(\mathrm{Lin}^{-} \mathrm{Sca}-1^{+} \mathrm{c}-\mathrm{Kit}^{+}\right)$, longterm HSC (LT-HSC), short-term HSC (ST-HSC), multipotent progenitor (MPP) and common myeloid progenitor in FA mice compared to WT (Figure 1D), although Fancg $^{-/}$and Fancc $^{-1}$; Fancg ${ }^{-/}$mice exhibited lower percentages of common lymphoid progenitors. Consistent with previous studies, ${ }^{5,11} \mathrm{HSC} /$ progenitors from $\mathrm{Fancc}^{-1} ; \mathrm{Fancg}^{-1}$ mice exhibited decreased clonogenic potential versus WT in colony-forming unit (CFU) assays (Figure 1E). In apop-

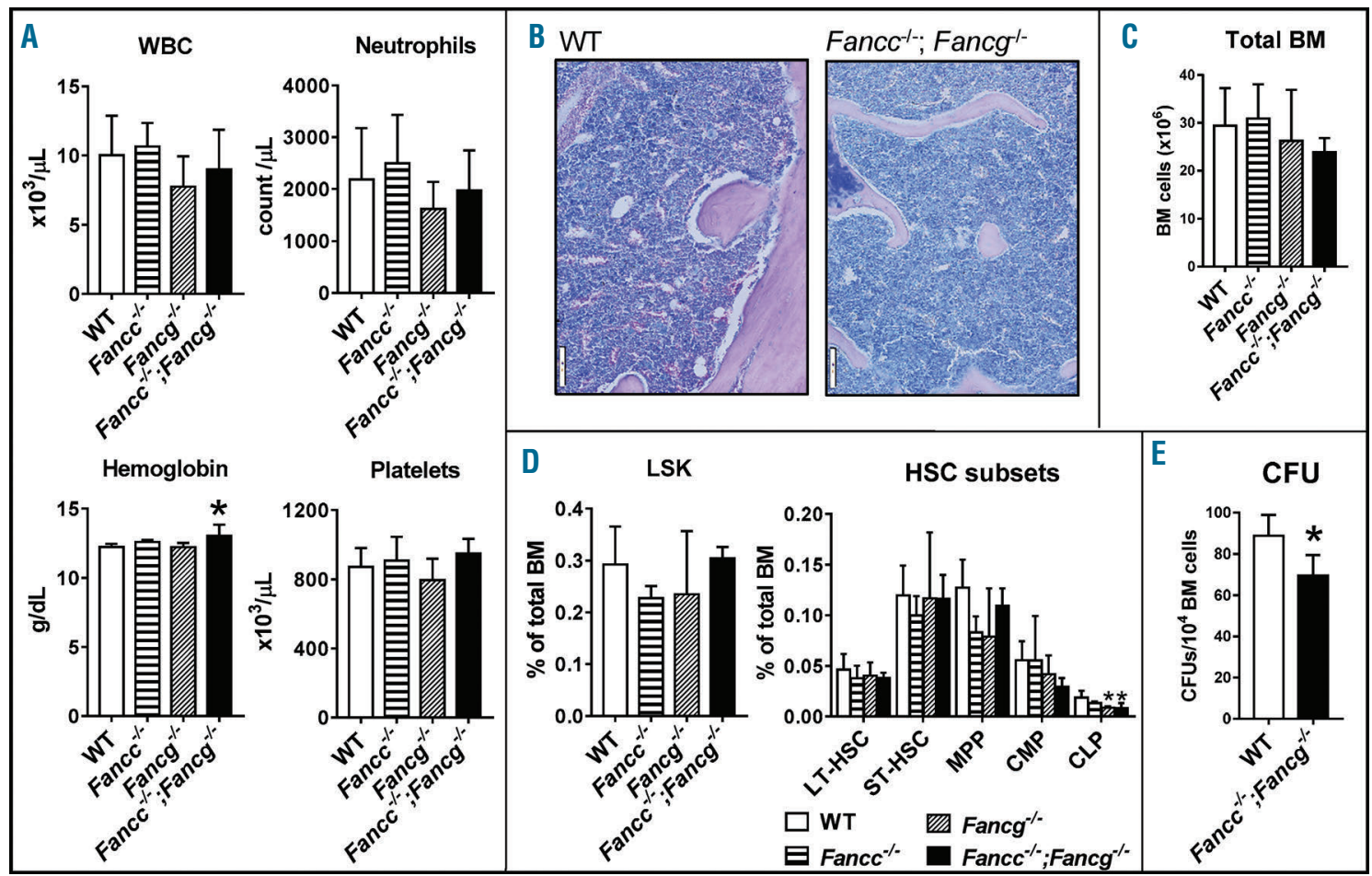

Figure 1. Mice with germline deficiency of Fancc and/or Fancg display normal peripheral blood counts, bone marrow (BM) cellularity and hematopoietic stem/progenitor cell frequencies. (A) Complete blood count (CBC) analysis of white blood cells (WBC), neutrophils, hemoglobin and platelets in WT, Fancc ${ }^{\prime}$, Fancs and Fancc ; Fancg mice. Mean \pm Standard Deviation (SD). N=4-8 mice per group. (B) Hematoxylin \& Eosin staining of BM section from a 6-month old wild-type (WT) and Fancc $\%$ Fancg double mutant mouse. (C) Total number of BM cells in 6-10-month old WT, Fancc $\%$ Fancg and FancC $\%$ Fancg mice

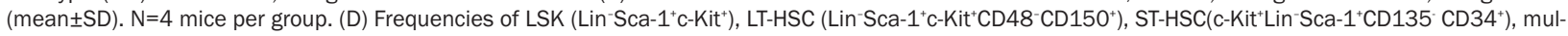
tipotent progenitor (MPP, Lin-Sca-1 $\left.1^{+} \mathrm{C}-\mathrm{Kit}^{+} \mathrm{CD} 135^{+}\right)$, common myeloid progenitor (CMP, Lin-Sca-1-C-Kit $\left.{ }^{+} \mathrm{CD} 34^{+}\right)$and common lymphoid progenitor $(\mathrm{CLP}$, Lin-Sca-1+C$\mathrm{Kit}^{+} \mathrm{CD} 127^{+}$) in 6-10-month old WT, Fancc $\%$, Fancg and Fancc $\%$ Fancg mice, expressed as the percentage of total BM cells (mean \pm SD). N=3-4 mice per group). (E) Total numbers of colony-forming units (CFU) per $10^{4} \mathrm{BM}$ cells from WT and Fancc $; F a n c{ }^{*}$ mice (mean $\left.\pm S D\right)$. N=4 mice per group. *P<0.05 compared with WT. 
tosis and cell cycling assays, we found that, compared to WT, Fancc and Fancc//Fanc ${ }^{-/ 1}$ mice possessed fewer CD48- LSK exhibiting early apoptosis (Annexin$\mathrm{V}^{+} 7 \mathrm{AAD}^{+}$) and an increased percentage of CD48- LSK from $\mathrm{Fancg}^{-/}$mice were in S/G2/M phase (Online Supplementary Figure $S 1 B$ and $C)$. A recent study demon- strated that polyinosinic-polycytidylic acid (pIpC) administration, which mimics viral infection through generating type I interferon responses, can induce BMF in Fanca ${ }^{-/}$mice. ${ }^{13}$ We injected pIpC into Fancc ${ }^{-1}$, Fance ${ }^{-/}$, and Fancc $^{-1}$; anc $^{-/}$mice. Compared with untreated or pIpCtreated WT, none of the pIpC-treated FA models devel-

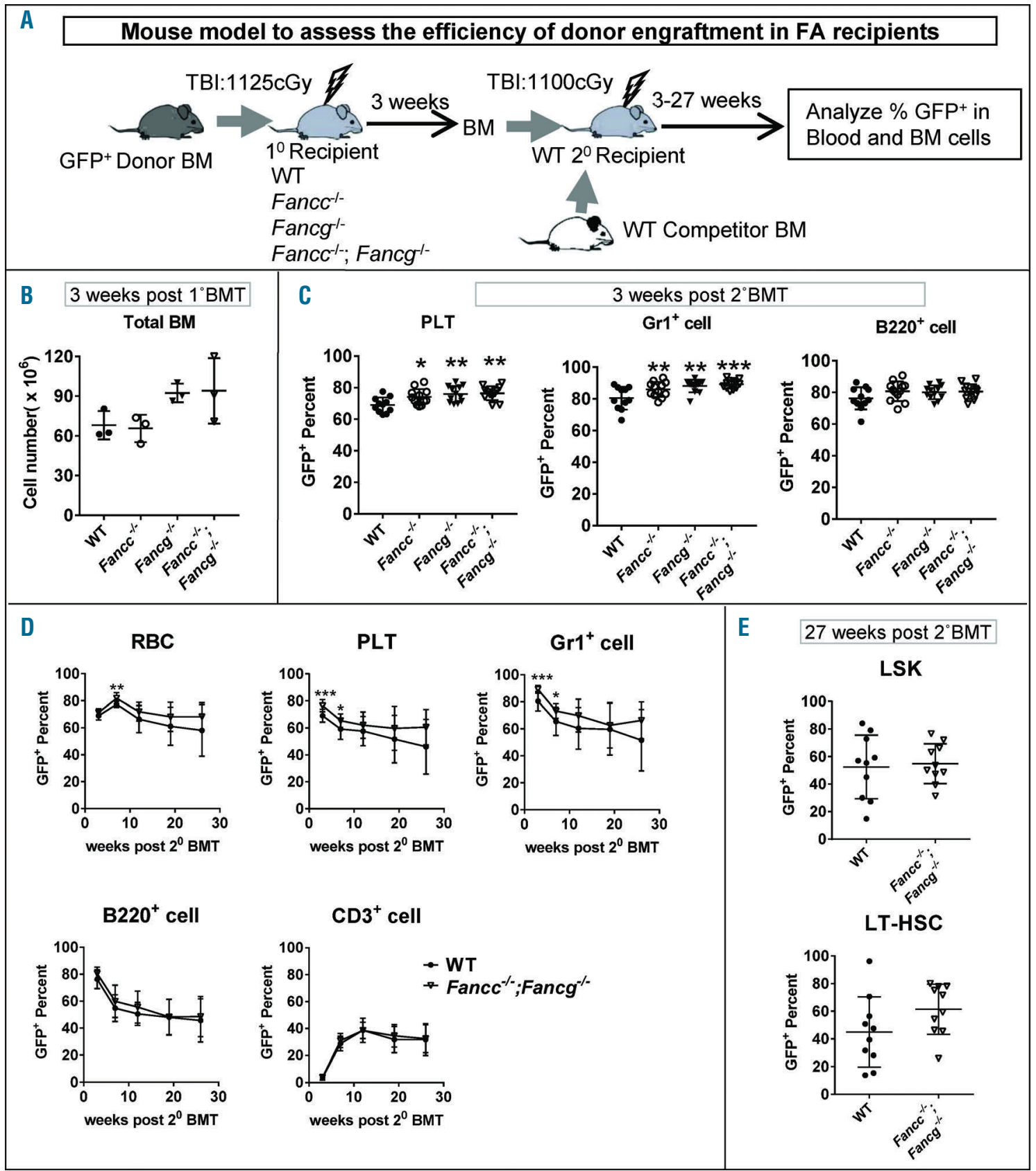

Figure 2. Fancc $\%$; Fancg double mutant mice display no reduction in the efficiency of long-term (LT)-hematopoietic stem cell (HSC) engraftment after HSC transplantation (HSCT) compared to wild-type (WT) controls, but show enhanced early hematopoietic progenitor engraftment. (A) Primary Fanconi anemia (FA) or WT recipients were transplanted with $5 \times 10^{6}$ whole bone marrow (BM) cells from H2K-GFP donor mice after receiving $1125 \mathrm{cGy}$ total body irradiation (TBI). The efficiency of GFP+ donor engraftment was assessed at three weeks after 1ry BM transplantation (BMT) using competitive secondary transplantation assay, in which equal volumes ( $20 \%$ of cumulative BM volume from bilateral hindleg bones) of whole BM from FA or WT 1ry recipients was transplanted with $10^{5}$ whole

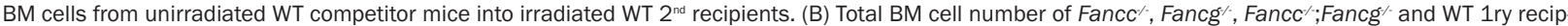
ients at three weeks after 1ry BMT. (C) Donor engraftment of early hematopoietic progenitors in WT and FA 1ry recipients examined by GFP ${ }^{+}$cell percentage in platelets (PLT), Gr1 myeloid cells and B220+ B cells in 2ry recipient mice at three weeks post 2ry BMT. (D) Assessment of donor HSC engraftment in WT and FA primary recipients by GFP ${ }^{+}$cell percentage in multiple mature blood lineage including red blood cells (RBC), PLT, Gr1 $1^{+}$myeloid cells, B220 ${ }^{+}$B cells and CD $3^{+}$

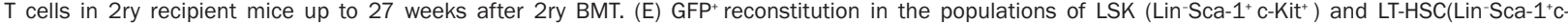
Kit $\left.{ }^{+} \mathrm{CD} 48-\mathrm{CD} 150^{+}\right)$in the $\mathrm{BM}$ of $2^{\text {nd }}$ recipient mice at 27 weeks after $2^{\text {nd }} \mathrm{BMT}$. ${ }^{*} P<0.05 ; * * P<0.01 ; * * * P<0.001$ compared with WT. 


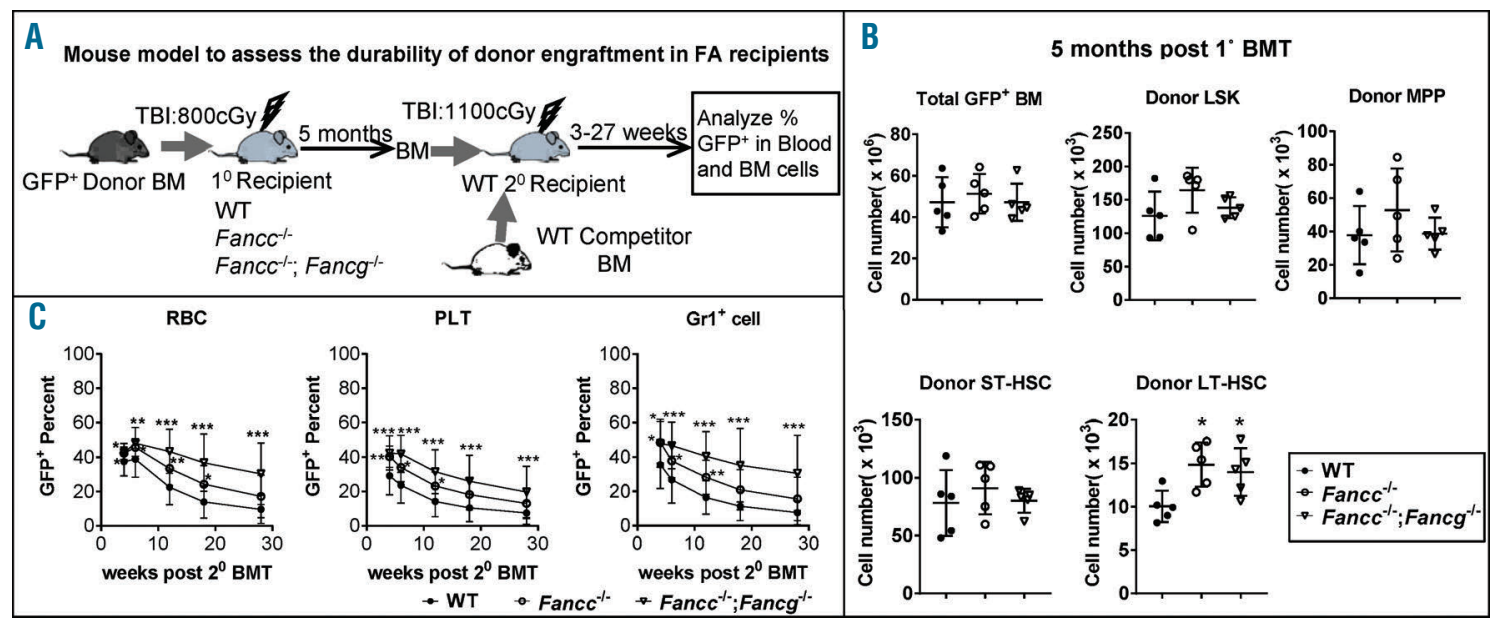

Figure 3. Mice with germline deficiency of FANCC and/or FANCG have durable hematopoietic stem cell (HSC) engraftment after transplantation. (A) Primary wild-type (WT) and Fanconi anemia (FA) recipients were transplanted with $5 \times 10^{6}$ whole bone marrow (BM) cells from H2K-GFP donor mice after receiving 800 cGy total body irradiation (TBI). Durability of $\mathrm{GFP}^{+}$donor engraftment was assessed at five months after 1ry BM transplantation (BMT) by competitive secondary transplantation assay, in which $10^{6}$ from each FA or WT 1ry recipients were transplanted with $5 \times 10^{5}$ whole BM cells from unirradiated WT mice into irradiated (1100cGy) WT 2ry recipients. (B) The BM of 1ry mice recipients were analyzed at five months after $1^{\text {st }}$ BMT. Scatterplots represent the cell cumber quantitation

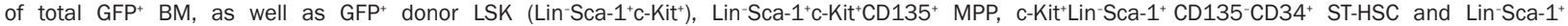

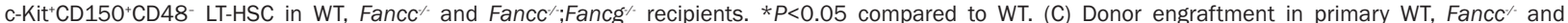
$\mathrm{Fancc}^{\prime} ;$ Fancg recipients was assessed by $\mathrm{GFP}^{+}$cell percentage in blood lineages including red blood cells (RBC), platelets (PLT) and Gr1 $1^{+}$myeloid cells in 2 ry recipient mice at 3-27 weeks after 2 ry BMT. $* P<0.05 ; * * P<0.01 ; * * * P<0.001$ compared with WT.

oped deficits in blood counts or BM cellularity (Online Supplementary Figure S1D and E).

Given the lack of appreciable BMF in Fancc, Fancg $^{-1}$ and $\mathrm{FancC}^{-1} ; \mathrm{Fancg}^{-/}$mice within the age ranges at which these mice would be used as HSCT recipients, we reasoned that we could use these models to test the impacts of FANCC and/or FANCG deficiency directly within mesenchymal niche cells on the capacity of the BM niche to facilitate efficient post-transplantation HSC engraftment, without the confounding influence of $\mathrm{BMF}$ on recipient niche cell function during HSCT. We thus transplanted $\mathrm{GFP}^{+}$WT whole BM into WT and $\mathrm{Fancc}^{-1}$; $\mathrm{Fancg}^{-/}$recipients two days after 1125 cGy total body irradiation (TBI). At three weeks post HSCT, we assessed $\mathrm{GFP}^{+} \mathrm{BM}$ reconstitution, finding that, rather than having impaired BM

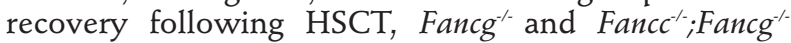
recipients exhibited a trend towards more rapid $\mathrm{BM}$ cell recovery ( $P=0.08$ and $P=0.14$, respectively) (Figure $2 \mathrm{~B}$ ). We then conducted competitive secondary (2ry) transplantation assays, in which the transplanted $\mathrm{GFP}^{+} \mathrm{BM}$ harvested from WT or FA recipients three weeks post-initial (1ry) HSCT were transplanted with $10^{5} \mathrm{BM}$ cells into irradiated secondary WT recipients (Figure 2A). At three weeks post-2ry BMT, $\mathrm{GFP}^{+}$reconstitution of myeloid $\left(\mathrm{GR} 1^{+}\right)$and platelet lineages, but not B lymphocytes $\left(\mathrm{B} 220^{+}\right)$, were increased in 2ry recipients of BM derived from FA versus WT primary recipients (Figure 2C), indicating that FA niches engrafted committed myeloid hematopoietic progenitor cells more efficiently than WT niches. To understand why FA niche leads to more rapid progenitor engraftment, we evaluated the expression of CXCL12, the chemokine critical in attracting HSC into $\mathrm{BM}$ niches. We found that residual BM stromal cells from irradiated Fancg $^{-/}$versus WT mice exhibited higher CXCL12 transcript expression (Online Supplementary Figure $S 2 B$ ). From 12-27 weeks post-2ry BMT, time points when hematopoiesis is driven specifically by donor LT-HSC derived from the WT competitor or primary recipient $\mathrm{BM}$, secondary recipients receiving WT and FA primary recipient $\mathrm{BM}$ showed similar $\mathrm{GFP}^{+}$reconstitution of blood cell lineages and BM LSK cells (Figure $2 \mathrm{D}$ and E, Online Supplementary Figure S2A and data not shown), indicating that FANCC and/or FANCG deficiency within HSCT recipient niche caused no deficits in donor HSC engraftment efficiency within the first three weeks post HSCT, even after myeloablative TBI. In fact, 2ry recipients receiving Fancc $^{-1}$; Fancg ${ }^{-/}$1ry recipient BM showed a trend toward higher $\mathrm{GFP}^{+}$reconstitution in $\mathrm{GR}^{+}$cells in blood $(P=0.08)$ and LT-HSC in BM $(P=0.11)$ than those receiving WT 1ry recipient BM (Figure $2 \mathrm{D}$ and E), which may reflect myeloid-bias propagated by FA niche. Taken together, compared to WT, BM niches in

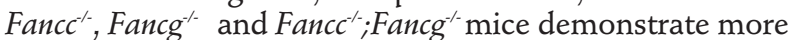
rapid $\mathrm{BM}$ reconstitution, enhanced committed progenitor engraftment, and no deficits in initial LT-HSC engraftment efficiency during the first three weeks post HSCT.

Next, we asked whether FANCC and/or FANCG deficiency in the BM niche impacted durable WT HSC engraftment post-HSCT. FA and WT 1ry recipients received $\mathrm{GFP}^{+} \mathrm{WT} \mathrm{BM}$ following a reduced-dose of 800 cGy-TBI due to concerns regarding long-term nonhematologic toxicity in the FA models. At five months post-1ry BMT, 1ry recipient BM was analyzed for immunophenotypic HSC/progenitors (Figure 3A). We found no reductions in total donor $\mathrm{BM}$ cell and any HSC/progenitor subsets in FA recipients versus WT recipients. In fact, all FA recipients displayed higher donor LTHSC numbers than WT recipients, and $\mathrm{Fancg}^{-/}$recipients also engrafted more $\mathrm{GFP}^{+} \mathrm{LSK}$ and ST-HSC (Figure 3B and Online Supplementary Figure $S 3 B-D)$. Functional HSC in 1 ry recipients was further analyzed by an additional competitive secondary transplantation assay. First recipient BM $\left(1 \times 10^{6}\right)$ together with $5 \times 10^{5}$ whole BM from unirradiated WT competitor mice was transplanted into irradiat- 
ed (1100 cGy) WT 2ry recipients (Figure 3A). Secondary recipients of FA versus WT 1ry recipient BM displayed no deficiency in $\mathrm{GFP}^{+}$reconstitution in multiple blood lineages between 3-27 weeks post-2ry BMT (Figure 3C and Online Supplementary Figure S3E). In fact, $\mathrm{GFP}^{+}$reconstitution of multilineage blood cells in 2ry recipients of

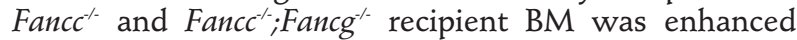
compared to those of WT recipient BM, though this enhancement may be caused by more residual host cells and less than complete $(80 \%) \mathrm{GFP}^{+}$reconstitution in WT 1ry recipients $\mathrm{BM}$ compared with $\mathrm{FA} 1^{\text {st }}$ recipients $\mathrm{BM}$ (Online Supplementary Figure S3A), due to the TBI dose used (800 cGy). Regardless, taken together, these data demonstrate that germline deficiency of FANCC and/or FANCG did not impair the durability of donor engraftment post HSCT.

We have demonstrated that germline deficiency of FANCC and/or FANCG did not impair the capacity of the $\mathrm{BM}$ niche to promote efficient and durable HSC engraftment post transplantation. Fancc ${ }^{-1}$, Fancg $^{--}$and Fancc $^{-1} ; \mathrm{Fancg}^{-/-}$mice demonstrated normal blood counts up to 40 weeks, in line with a previous study of Fancg mice. ${ }^{12}$ We also found no deficits in BM cellularity or immunophenotypic HSC/progenitors in mice with FANCC and/or FANCG deficiency even with pIpCinduced HSC cycling. However, $\mathrm{Fancc}^{-1} ; \mathrm{Fancg}^{-/} \mathrm{HSC} /$ progenitors showed reduced clonogenic potential consistent with previous reports. ${ }^{5,11}$ Comparing efficiency and durability of HSC engraftment in WT versus FA HSCT recipients by assessing functional HSC content at three weeks and five months post HSCT through competitive secondary transplantation, we found no deficiency in HSC engraftment in FA recipients. Previously, Zhou et al. suggested that $\mathrm{Fancc}^{-1}$; Fancg ${ }^{-/}$recipients have decreased nichemediated donor HSC engraftment, based on their observation of decreased CFU number in BM after transplantation with WT BM. ${ }^{5}$ It is possible that this decreased CFU number was relative, and could be explained by the more rapid $\mathrm{BM}$ reconstitution and increased hematopoietic progenitor engraftment seen in FA recipients initially following HSCT, which could lead to true colony-forming HSC representing a lower percentage of BM cells at the time studied. The higher CXCL12 expression we identified in the irradiated Fanco ${ }^{-/}$BM environment may explain why FA recipients have increased hematopoietic progenitor engraftment/expansion initially after HSCT, though further comparisons of molecular and cellular profiles within FA versus WT recipient BM niches are needed.

While survival rates of HSCT in FA have improved over the last two decades, strategies to further reduce conditioning intensity could reduce complications and improve quality of life. ${ }^{14} \mathrm{BM}$ niche dysfunction, either due to preexisting $\mathrm{BMF}$ or the direct impact of FA gene deficiency on niche cells poses a theoretical obstacle to stable engraftment with further reduced conditioning intensity for HSCT in FA. Our study has demonstrated for the first time that the functional capacity of recipient $\mathrm{BM}$ niche to stably engraft donor HSC remains intact despite FANCC and/or FANCG deficiency within the niche. To determine whether BMF itself impacts niche function during HSCT for FA, new FA models in which severe BMF can reproducibly be induced are required. One such approach could be to generate humanized BM niche xenografts by transplanting FA patient-derived MSPC and HSC/progenitors into immunodeficient mice, as previously described. ${ }^{15}$
Ji Zha, Lori Kunselman, Jian-Meng Fan, ${ }^{1}$ and Timothy S. $\mathrm{Olson}^{1,2,3^{*}}$

'Comprehensive Bone Marrow Failure Center, Children's Hospital of Philadelphia, Philadelphia; ${ }^{2}$ Blood and Marrow Transplant Section, Division of Oncology, Department of Pediatrics, Children's Hospital of Philadelphia, Philadelphia and ${ }^{3}$ Perelman School of Medicine,

University of Pennsylvania, Philadelphia, PA, USA

Acknowledgments: we gratefully acknowledge Dr. Feng-Chun Yang and Dr. Wade Clapp (Indiana University School of Medicine, Indianapolis, IN) for providing Fancc- ${ }^{-1}$ Fancg-r mice. $^{-1}$

Funding: the funding of this work is supported by National Institutes of Health K08 HL122306 (TSO), American Society of Hematology Scholar Award (TSO) and WW Smith Charitable Trust.

Correspondence: TIMOTHY S OLSON.

olsont@email.chop.edu.

doi:10.3324/haematol.2018.202143

Information on authorship, contributions, and financial \& other disclosures was provided by the authors and is available with the online version of this article at www. haematologica.org.

\section{References}

1. Hooper AT, Butler JM, Nolan DJ, et al. Engraftment and Reconstitution of Hematopoiesis Is Dependent on VEGFR2Mediated Regeneration of Sinusoidal Endothelial Cells. Cell Stem Cell. 2009;4(3):263-274.

2. Caselli A, Olson TS, Otsuru S, et al. IGF-1-mediated osteoblastic niche expansion enhances long-term hematopoietic stem cell engraftment after murine bone marrow transplantation. Stem Cells. 2013;31(10):2193-2204.

3. Olson TS, Caselli A, Otsuru S, et al. Megakaryocytes promote murine osteoblastic HSC niche expansion and stem cell engraftment after radioablative conditioning. Blood. 2013;121(6):5238-5249.

4. Morrison SJ, Scadden DT. The bone marrow niche for haematopoietic stem cells. Nature. 2014;505(7483):327-334.

5. Zhou Y, He Y, Xing W, et al. An abnormal bone marrow microenvironment contributes to hematopoietic dysfunction in fanconi anemia. Haematologica. 2017;102(6):1017-1027.

6. Zambetti NA, Ping Z, Chen S, et al. Mesenchymal inflammation drives genotoxic stress in hematopoietic stem cells and predicts disease evolution in human pre-leukemia. Cell Stem Cell. 2016; 19(5):613-627.

7. Raaijmakers MHGP, Mukherjee S, Guo S, et al. Bone progenitor dysfunction induces myelodysplasia and secondary leukaemia. Nature. 2010;464(7290):852-857.

8. Río P, Navarro S, Bueren JA. Advances in Gene Therapy for Fanconi Anemia. Hum Gene Ther. 2018;29(10):1114-1123.

9. Kuehl J, Pickel S, Xue Y, et al. Biallelic mutations in FAAP100 predispose to Fanconi anemia (FA). In: 30th Fanconi Anemia Research Fund Scientific Symposium. Newport Beach. 2018:26.

10. Adam S, Melguizo Sanchis D, El-Kamah G, et al. Concise Review: Getting to the Core of Inherited Bone Marrow Failures. Stem Cells. 2017;35(2):284-298

11. Pulliam-Leath AC, Ciccone SL, Nalepa G, et al. Genetic disruption of both Fancc and Fancg in mice recapitulates the hematopoietic manifestations of Fanconi anemia. Blood. 2010;116(16):2915-2920.

12. Barroca V, Mouthon MA, Lewandowski D, et al. Impaired functionality and homing of fancg-deficient hematopoietic stem cells. Hum Mol Genet. 2012;21(1):121-135

13. Walter D, Lier A, Geiselhart A, et al. Exit from dormancy provokes DNA-damage-induced attrition in haematopoietic stem cells. Nature. 2015;520(7548):549-552.

14. MacMillan ML, Wagner JE. Haematopoeitic cell transplantation for Fanconi anaemia - When and how? Br J Haematol. 2010;149(1):1421.

15. Reinisch A, Thomas D, Corces MR, et al. A humanized bone marrow ossicle xenotransplantation model enables improved engraftment of healthy and leukemic human hematopoietic cells. Nat Med. 2016;22(7):812-821. 